\title{
Los derechos humanos en la opinión pública salvadoreña
}

\author{
Instituto Universitario de Opinión Pública
}

\begin{abstract}
Resumen
Entre el 11 y el 19 de marzo de 1995, el IUDOP con la colaboración del Instituto de Derechos Humanos de la UCA realizó un sondeo de opinión pública para conocer la opinión de los salvadoreños sobre la situación de los derechos humanos en El Salvador y para establecer los principales retos del trabajo de su protección. Los resultados mostraron que a pesar de que la población piensa que todavía no existe un pleno respeto de los derechos humanos en el país, tampoco existe un pleno conocimiento del tema. La mayoria de ciudadanos relaciona de alguna manera el problema del respeto de los derechos humanos con el auge de la delincuencia que vive el país.
\end{abstract}

\section{Encuesta y población encuestada}

El sondeo para recoger la opinión de los salvadoreños sobre los derechos humanos, para evaluar su situación en El Salvador y para definir las principales tareas de la Procuradora para la Defensa de los Derechos Humanos, fue llevada a cabo entre el 11 y el 19 de marzo del año en curso. Esta constituye la primera evaluación sobre derechos humanos que se proyecta realizar en el curso de 1995 y es consecuencia de una tarea de vigilancia después de la salida de ONUSAL, en el primer cuatrimestre del año. El cuestionario de la encuesta fue preparado con la colaboración del Instituto de Derechos Humanos de la UCA (IDHUCA) e intentaba recoger opiniones sobre distintos tópicos relacionados con el problema de los derechos humanos en El Salvador.

La boleta se componía de cuatro grandes partes. La primera, como ya es costumbre, recogía los datos demográficos de las personas: sexo, edad, escolaridad, condición de empleo y religión; además de aquella información llenada por el encuestador, referida a ciertas condiciones del entrevistado: estrato socioeconómico, departamento y municipio de vivienda. La segunda parte del cuestionario estaba constituida brevemente por tres preguntas que exploraban el conocimiento de la población sobre el tema de los derechos humanos: qué son los derechos humanos, cuáles son los derechos humanos más importantes y el conocimiento acerca de la Declaración Universal de los Derechos Humanos. La sección número tres del cuestionario contenía una serie de diez reactivos en una escala de acuerdo-desacuerdo, referida a valores y opiniones sobre la situación de los derechos humanos en el país, los derechos laborales y las responsabilidades del gobierno en el respeto por los derechos fundamentales de la persona. La cuarta parte de la boleta de investigación evaluaba 
el quehacer de las instituciones, cuya responsabilidad tiene que ver con la vigilancia de los derechos ciudadanos: la Procuraduría para la Defensa de los Derechos Humanos, la Corte Suprema de Justicia y la Policía Nacional Civil; en concreto, la primera mereció una batería de preguntas de carácter evaluativo más algunas otras que recogían las expectativas de los salvadoreños sobre el trabajo de la dirección del organismo. Finalmente, el cuestionario cerraba con una serie de casi trece preguntas con tópicos relacionados siempre con el tema de los derechos humanos: impunidad en El Salvador, evaluación de ONUSAL, manifestaciones y desestabilización, papel de la Fuerza Armada, etc.

Una vez preparada la boleta para el sondeo, se diseñó una muestra nacional que reflejase lo más fielmente posible la totalidad de la población salvadoreña; esto según los datos del censo nacional de población de 1992 y los últimos reportes de la Encuesta de Hogares del Ministerio de Planificación. La muestra total incluyó diez de los catorce departamentos de la república: Santa Ana, Sonsonate, La Libertad, San Salvador, Cabañas, San Vicente, Usulután, San Miguel, Morazán y La Unión. En cada departamento, la muestra se divi- dió en dos grandes sectores: urbano y rural. El primero fue estratificado en cinco estratos: alto, medio-alto, medio-bajo, obrero y marginal. El sector rural se dejó como categoría única por las dificultades para encontrar criterios de tipificación o estratificación en el campo. En el muestreo del país se consideraron también cuotas para los rangos de edad y para el género de los encuestados.

La aplicación del cuestionario se hizo por aproximación no sistemática a los hogares ubicados en los municipios y en las zonas definidas aleatoriamente. Los entrevistadores explicaban a las personas abordadas los objetivos y el tema de la encuesta. En cada caso se entrevistó únicamente a personas que quisieran contestar (una persona por hogar) y que cumplieran con los requerimientos descritos en la boleta para completar la cuota muestral.

La muestra final obtenida fue de 1,153 encuestas válidas con un margen de error estimado del +1- 0.04 (cuatro por ciento). En el Cuadro 1 se presenta la distribución de la población encuestada según departamento de vivienda y el estrato socioeconómico al que pertenece.

\section{Cuadro 1}

\section{Población encuestada por departamento y sector social}

\begin{tabular}{|c|c|c|c|c|c|c|c|c|}
\hline \multirow{2}{*}{ Departamento } & \multicolumn{6}{|c|}{ Sector social } & \multicolumn{2}{|c|}{ Todos } \\
\hline & $\overline{\text { Alto }}$ & Med-al & Med-baj & Obrero & Marginal & Rural & $\mathbf{N}$ & $\%$ \\
\hline Santa Ana & 10 & 16 & 26 & 23 & 18 & 61 & 154 & 13.4 \\
\hline Sonsonate & 2 & 17 & 12 & 19 & 12 & 48 & 110 & 9.5 \\
\hline La Libertad & 7 & 16 & 17 & 24 & 16 & 58 & 138 & 12.0 \\
\hline San Salvador & 35 & 30 & 68 & 76 & 68 & 58 & 335 & 29.1 \\
\hline Cabañas & 4 & 7 & 7 & 12 & 7 & 42 & 82 & 6.9 \\
\hline San Vicente & 5 & 8 & 8 & 11 & 6 & 43 & 81 & 7.0 \\
\hline Usulután & 4 & 7 & 9 & 11 & 8 & 27 & 66 & 5.7 \\
\hline San Miguel & 5 & 15 & 15 & 20 & 10 & 24 & 89 & 7.7 \\
\hline Morazán & 1 & 5 & 6 & 5 & 4 & 21 & 42 & 3.6 \\
\hline La Unión & 5 & 6 & 6 & 10 & 5 & 27 & 59 & 5.1 \\
\hline Todos $\mathrm{N}$ & 78 & 127 & 174 & 211 & 154 & 409 & 1153 & - \\
\hline$\%$ & 6.8 & 11.0 & 15.1 & 18.3 & 13.4 & 35.5 & - & 100 \\
\hline
\end{tabular}


El 51.8 por ciento de las personas encuestadas pertenece al género femenino, mientras que el 48.2 por ciento son hombres. La edad promedio es de 36.07 años con una desviación típica de 13.5 años; la mayor parte de los salvadoreños entrevistados se concentran en el rango que va desde los 26 a los 40 años de edad. El Cuadro 2 muestra la distribución de los encuestados de acuerdo al nivel de estudios alcanzado; según éste, casi la mitad de los ciudadanos no ha cursado estudios más allá de primaria o sexto grado, mientras que sólo el 15 por ciento ha llegado hasta la universidad. De hecho, el promedio de años estudiados para la población total es de 7.4 años de educación, con una desviación típica de 4.8 años. En otro orden, el Cuadro 3 revela la proporción de salvadoreños que se declaran empleados y desempleados en función del sector socioeconómico al que pertenecen. Este cuadro muestra una clara relación entre la condición de empleo de la personas encuestadas y su pertenencia a determinado estrato: en la medida en que se "desciende" en la escala socioeconómica, la proporción de las personas sin empleo es mayor, alcanzando alrededor del 50 por ciento en los sectores marginales y rurales. Obviamente, tener empleo todavía parece ser un privilegio de ciertos estratos.

\section{Cuadro 2}

Distribución de la población encuestada según nivel de estudios

\begin{tabular}{lrc}
\hline Nivel educativo & $\mathrm{N}$ & Porcentajes \\
\hline Ninguno & 163 & 14.1 \\
Primaria & 376 & 32.6 \\
Básico & 181 & 15.7 \\
Bachillerato & 208 & 18.0 \\
Superior no univ. & 45 & 3.9 \\
Universitario & 172 & 14.9 \\
No responde & 8 & 0.7 \\
\hline
\end{tabular}

Finalmente, otro dato demográfico recogido por la encuesta muestra que, en términos religiosos, la mayoría de los salvadoreños sigue profesando la fe católica, esto es, dos de cada tres salvadoreños (el 65 por ciento); sin embargo, la restante tercera parte se divide en dos grupos: los lla- mados evangélicos, con el 14.5 por ciento de los encuestados y las personas que dicen no seguir a ninguna confesión religiosa, con el 17.2 por ciento. Pequeños porcentajes se reparten en otras confesiones religiosas.

Cuadro 3

Condición de empleo según sector social de los encuestados

(En porcentajes)

\begin{tabular}{lcc}
\hline \multirow{2}{*}{ Estrato } & \multicolumn{2}{c}{ Condición } \\
\cline { 2 - 3 } & Empleado & Desempleado \\
\hline Alto & 75.6 & 24.4 \\
Medio-alto & 66.1 & 33.9 \\
Medio-bajo & 64.9 & 35.1 \\
Obrero & 64.5 & 35.5 \\
Marginal & 48.7 & 51.3 \\
Rural & 51.1 & 48.9 \\
Todos & 58.6 & 41.4 \\
\hline
\end{tabular}

$\mathrm{X}^{2}=33.88 ; \mathrm{g} .1 .=5 ; \mathrm{p}=0.0000$

2. El conocimiento sobre los derechos humanos

Solamente uno de cada cuatro salvadoreños (26.5 por ciento) ha oído hablar de la Declaración Universal de los Derechos Humanos. Esto puede ofrecer desde ya una idea de la importancia que en este país se ha dado a la educación y a la práctica de los derechos humanos. Este nivel de conocimiento de la Declaración está directamente relacionado con dos variables demográficas: el estrato socioeconómico y el nivel educativo de los encuestados. Precisamente, los más pobres y de menor educación son quienes ignoran en mayor proporción la existencia de tal Declaración —en el área rural sólo la conoce el 15 por ciento.

Preguntados sobre el concepto de derechos humanos, la tercera parte (el 33 por ciento) los definió como respeto; mientras que el 20.4 por ciento los concibe como "aquellos derechos o privilegios que poseemos como personas"; y el 16.2 por ciento los delimitó como "algo que nos protege". Porcentajes menores definían los derechos humanos como derecho a la libertad ( 6.4 por ciento) y satis- 
facción de necesidades básicas (3.6 por ciento), entre otras respuestas. Sin embargo, llama la atención de que cerca de un 20 por ciento de los encuestados no supo dar una definición sobre los derechos humanos. Un análisis por sectores sociales mostró que en los sectores medios y altos urbanos se destaca la definición de respeto y el carácter intrísecamente humano de los derechos, mientras que en los estratos más pobres de la población —obreros, marginales y campesinos - la concepción sobre los derechos humanos se centra en la función protectora de los mismos

Ligado a lo anterior, el sondeo realizado por el IUDOP pidió a los ciudadanos que mencionaran los derechos humanos que consideraban más importantes. Examinando las respuestas sobre el primer derecho humano mencionado, se puede ver que los derechos que más valora la población constituyen el conjunto de facultades básicas: el respeto a la vida y la libertad. Juntas, estas respuestas reúnen casi al 60 por ciento de las referencias de los ciudadanos consultados. Esto es lógico si se toma en cuenta la precariedad de la cultura de derechos humanos que ha existido en este país durante su historia. En unas circunstancias sociales en las que la vida resulta tan incierta y las libertades ciudadanas son todavía una novedad producto de los acuerdos de paz, los salvadoreños citan precisamente los derechos que más se han violado en el país

\section{Opiniones y valores sobre los derechos hu- manos}

El sondeo en cuestión incluyó una parte en que se presentaban una serie de afirmaciones frente a las cuales los encuestados debían revelar su opinión, mostrándose de acuerdo o en desacuerdo. Tales afirmaciones tocaban temas relacionados con la justicia social en el país, el interés del gobierno por los derechos de los ciudadanos, la relación entre los derechos humanos y la delincuencia y las condiciones de respeto a los derechos laborales. En el Cuadro 5 se presentan los resultados de esta batería, según la temática de cada afirmación.

\section{Cuadro 4}

Los derechos humanos más importantes

(En porcentajes)

\begin{tabular}{|c|c|c|c|c|c|c|c|}
\hline \multirow[b]{2}{*}{ Derechos } & \multicolumn{6}{|c|}{ Sector social } & \multirow[b]{2}{*}{ Todos } \\
\hline & Alto & Med-al & Med-baj & Obrero & Marginal & Rural & \\
\hline Respeto a la vida & 29.5 & 40.2 & 36.2 & 28.0 & 22.1 & 30.1 & 30.6 \\
\hline Libertad & 26.9 & 22.8 & 21.8 & 15.2 & 12.3 & 9.0 & 15.3 \\
\hline Respeto & 15.4 & 9.4 & 10.9 & 11.4 & 14.3 & 10.5 & 11.4 \\
\hline Derecho a la salud & 3.8 & 6.3 & 10.3 & 14.2 & 12.3 & 10.8 & 10.6 \\
\hline Protección al niño & 11.5 & 13.4 & 10.9 & 11.8 & 10.4 & 7.8 & 10.2 \\
\hline Derecho al trabajo & 3.8 & 2.4 & 2.3 & 7.1 & 5.8 & 8.6 & 6.0 \\
\hline Seguridad pública & 3.8 & 1.6 & 1.1 & 2.8 & 1.9 & 2.0 & 2.1 \\
\hline Otras respuestas & 2.6 & 1.6 & 3.4 & 1.4 & 3.8 & 2.4 & $2.5^{\circ}$ \\
\hline No sabe & 2.6 & 2.4 & 2.9 & 8.1 & 16.9 & 18.8 & 11.3 \\
\hline
\end{tabular}

Ahora dígame por favor los dos derechos humanos que sean más importantes para usted. (Se muestran los resultados del primer derecho humano mencionado.) 


\section{Cuadro 5 \\ Opiniones hacia algunas afirmaciones sobre derechos humanos \\ (En porcentajes)}

\begin{tabular}{|c|c|c|c|c|}
\hline \multirow[b]{2}{*}{ Afirmaciones } & \multicolumn{4}{|c|}{ Opinión } \\
\hline & De acuerdo & Medio & Desacuerdo & No sabe \\
\hline \multicolumn{5}{|l|}{ Justicia social y desempeño del Estado } \\
\hline En nuestro país existe un régimen de justicia social & 41.1 & 2.6 & 51.0 & 5.3 \\
\hline El gobierno se preocupa por los DDHH de todos los salvadoreños & 35.1 & 2.7 & 58.8 & 3.4 \\
\hline Hasta la fecha el gobierno ha estado al servicio de unos pocos & 79.5 & 1.4 & 14.9 & 4.2 \\
\hline \multicolumn{5}{|l|}{ Derechos laborales } \\
\hline $\begin{array}{l}\text { Es muy importante afiliarse o formar parte de una organización } \\
\text { para defender los propios derechos }\end{array}$ & 75.2 & 1.4 & 18.0 & 5.3 \\
\hline $\begin{array}{l}\text { En las fábricas maquiladoras extranjeras se violan los derechos } \\
\text { de los trabajadores }\end{array}$ & 66.4 & 1.0 & 7.1 & 25.4 \\
\hline $\begin{array}{l}\text { Las manifestaciones callejeras sólo consiguen armar alboroto y } \\
\text { crear problemas sociales y políticos }\end{array}$ & 62.9 & 2.5 & 29.6 & 5.0 \\
\hline \multicolumn{5}{|l|}{ Derechos humanos y delincuencia } \\
\hline $\begin{array}{l}\text { puede acabar con ellos } \\
\text { Ya que el gobierno no proporciona justicia o seguridad, la }\end{array}$ & 61.2 & 1.6 & 29.5 & 8.0 \\
\hline $\begin{array}{l}\text { gente tiene el derecho de buscar la justicia por su propia mano } \\
\text { En este país, los organismos de los derechos humanos sólo }\end{array}$ & 53.1 & 0.9 & 43.6 & 2.4 \\
\hline buscan proteger a los delincuentes & 43.1 & 2.4 & 46.3 & 8.2 \\
\hline $\begin{array}{l}\text { Situación de derechos humanos y acuerdos de paz } \\
\text { La situación de los derechos humanos ha mejorado claramente } \\
\text { desde la firma de los acuerdos de paz }\end{array}$ & 62.3 & 4.6 & 29.9 & 3.2 \\
\hline
\end{tabular}

A continuación, le voy a leer una serie de afirmaciones sobre distintos temas relacionados con los derechos humanos, dígame por favor si está de acuerdo, algo de acuerdo, algo en desacuerdo o en desacuerdo.

Las opiniones que se muestran en el rubro de justicia social y desempeño del Estado revelan una valoración negativa acerca de las condiciones de justicia en el país y el trabajo del Estado. No sólo la mitad de los salvadoreños no comparten la idea de que en el país exista un régimen de justicia social, sino que la mayoría piensa que el gobierno no se preocupa por los derechos humanos de todos los salvadoreños rematada por la opinión de que el Estado ha estado al servicio de unos pocos.

La parte de derechos laborales resulta bastante interesante. Tres de cada cuatro salvadoreño están de acuerdo con que es importante organizarse para defender sus derechos; sin embargo, dos de cada tres consideran que las manifestaciones sólo consiguen crear problemas sociales. Aparentemente, las manifestaciones públicas han adquirido una mala imagen entre la población, no así las iniciativas de organización para defender los propios derechos, aunque según las encuestas anteriores son muy pocos los ciudadanos que admiten estar organizados (ver $E C A, 1994,545-546$ ). En esta batería, la encuesta indagó también la opinión de los salvadoreños sobre la situación de las obreras en las maquilas: dos de cada tres salvadoreños comparten la opinión de que en las fábricas maquiladoras extranjeras se violan los derechos de las trabajado- 
ras. Esta opinión llega a ser sostenida por ocho de cada diez personas dentro del estrato obrero.

Probablemente, uno de los hallazgos más interesantes de esta investigación de opinión pública es el que se refiere a la relación entre los derechos humanos y la delincuencia. Existe una importante proporción de salvadoreños que culpan a los derechos humanos de la ineficiencia en el combate de la delincuencia. Para casi dos terceras partes de la población, los derechos humanos favorecen a los delincuentes y así no se puede acabar con ellos. Esta postura es sostenida con mayor frecuencia entre los estratos socioeconómicamente más prósperos que entre los sectores pobres. Paradójicamente con lo que se podría esperar, los ciudadanos con mayores niveles de educación son también los más propensos en ver los derechos humanos de manera peyorativa. Sin embargo, al pedir la postura sobre el papel de los organismos protectores de los derechos humanos en relación a la criminalidad, las opiniones parecen más divididas. Mientras que el 43 por ciento de los ciudadanos considera que las organizaciones no gubernamentales de derechos humanos sólo buscan proteger a los delincuentes, el 46 por ciento se muestra en desacuerdo con esa idea. Esto mostraría un poco de cautela de parte de la población respecto a emitir opiniones que resulten más comprometedoras. De hecho, sólo un poco más de la mitad de los encuestados estaría de acuerdo con que la gente tome la justicia por su propia mano, aunque hay que reconocer que, de suyo, la proporción sigue siendo grande. Estos resultados revelan en términos generales una interpretación sesgada del concepto de derechos humanos entre buena parte de la población. Es probable que tal interpretación venga del manejo que sobre el problema de la delincuencia hacen algunos funcionarios públicos cuando acusan a la observación de ciertos procedimientos como una de las causas de la ineficiencia en el combate al crimen; por otro lado, el mismo auge delincuencial y la desesperación de los ciudadanos por la falta de soluciones explica, hasta cierto punto, la indiferencia hacia aquellos derechos fundamentales para reclamar medidas más drásticas y autoritarias.

Finalmente, el Cuadro 5 muestra que para el 62.3 por ciento de la gente, la situación de respeto de los derechos humanos ha mejorado claramente desde la firma de los acuerdos de paz. Esta opinión es sostenida con mayor frecuencia entre la gente de clase alta y del estrato rural, y entre los que poseen menores años de formación educativa.

\section{Las instituciones que defienden los derechos humanos}

De acuerdo a la encuesta de marzo, los salvadoreños eligieron a las organizaciones no gubernamentales, a la Procuraduría para la Defensa de los Derechos Humanos, a la Policía Nacional Civil y a ONUSAL, entre otras, como las instituciones que más defienden los derechos humanos de la población. Estas respuestas muestran tendencias que es importante comentar. En primer lugar, evaluaciones hechas en los años pasados habian mostrado siempre a ONUSAL con el mayor porcentaje de elección como la institución que defendía los derechos humanos de la población; de hecho, mucha gente definía a ONUSAL como la primera institución a donde iŕa a poner una denuncia si sufriera un percance. Este sondeo revela que el perfil en derechos humanos de ONUSAL ha sido superado por el de varias instituciones. En segundo lugar y relacionado con lo anterior, la subida de la Policía Nacional Civil, que es muy significativa, podría estar relacionada con el papel cada vez más

\section{Instituciones que defienden más los derechos humanos}

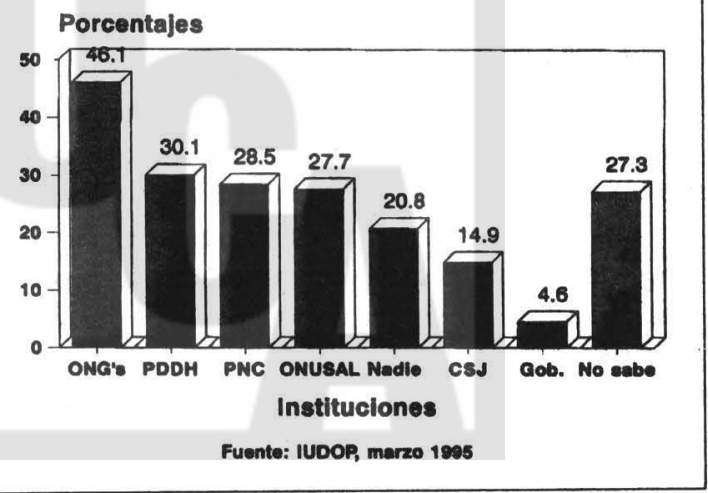

Figura 1. ¿Podrla decirme las dos instituciones que a su juicio defienden mejor los derechos humanos en nuestro país? (Se incluyen las dos opciones) 
protagónico que juega en el combate de la delincuencia. Si los derechos más importantes para buena parte de la población están relacionados con el respeto a la vida y a la integridad física, es lógico que una de las instituciones mejor vistas en el respeto de los derechos humanos sea la que combate al causante del irrespeto a la vida y a la integridad física.

En tercer lugar, la mención de la Procuraduría para la Defensa de los Derechos Humanos como una de las principales instituciones que defienden los derechos del ciudadano es, en sí misma, un avance significativo del perfil de esta institución. Aunque sería más lógico esperar que este organismo figurase en el primer lugar de las respuestas por la naturaleza de su mandato. Con todo, el resultado más llamativo lo constituye la aparición de las organizaciones no gubernamentales en el primer lugar de las respuestas. Las organizaciones mencionadas como defensoras de los derechos humanos son esencialmente tres: la Comisión de Derechos Humanos No Gubernamental, Tutela Legal del Arzobispado y el Instituto de Derechos Humanos de la UCA. De ellas, Tutela Legal es la que obtiene los reconocimientos mayores por parte de la población. Este resultado probablemente estaría indicando la creciente confianza de los salvadoreños en instituciones que provienen de la sociedad civil como resultado, por otro lado, del desgaste de las instituciones de la llamada sociedad política.

En los subsiguientes apartados se expone la opinión pública referida a cada una de las instituciones comprometidas en el respeto de los derechos humanos: La Procuraduría para la Defensa de los Derechos Humanos, la Policía Nacional Civil, ONUSAL y la Corte Suprema de Justicia.

\subsection{La Procuraduría para la Defensa de los De- rechos Humanos}

Precisamente, uno de los propósitos del sondeo de marzo era evaluar el trabajo de la Procuraduría de Derechos Humanos y recoger las expectativas de la población sobre su trabajo de cara a la elección de la nueva directora de la institución. En tal sentido, la encuesta dedicaba buena parte de sus preguntas a esta institución. La batería comenzaba preguntando a las personas si conocían la existencia de la Procuraduría. Los resultados son muy elocuentes.

\section{Cuadro 6 \\ Conocimiento sobre la Procuraduría para la Defensa de los Derechos Humanos según estrato y nivel educativo (En porcentajes)}

\begin{tabular}{ccc}
\hline \multirow{3}{*}{ Variables } & \multicolumn{2}{c}{ Conocimiento } \\
\cline { 2 - 3 } & No & Sí \\
\hline
\end{tabular}

\section{Estrato}

\begin{tabular}{|l|l|l|}
\hline Alto & 23.1 & 76.9 \\
\hline Medio-alto & 27.6 & 72.4 \\
\hline Medio-bajo & 31.0 & 69.0 \\
\hline Obrero & 44.1 & 55.9 \\
\hline Marginal & 48.1 & 51.9 \\
Rural & 60.1 & 39.9 \\
& & \\
& Estudios & \\
\hline Ninguno & 66.3 & 33.7 \\
Primaria & 56.1 & 43.9 \\
Plan básico & 42.5 & 57.5 \\
Bachillerato & 33.2 & 66.8 \\
Superior & 23.5 & 76.5 \\
\hline Todos & 45.1 & 54.9 \\
\hline
\end{tabular}

¿Ha oído Ud. hablar de la Procuraduría para la Defensa de los Derechos Humanos?

Casi la mitad de los ciudadanos salvadoreños no ha oído hablar acerca de la Procuraduría. Este porcentaje es significativamente mayor entre las personas de sectores descendentes económicamente $y$ entre aquellos de menor nivel educativo. Paradójicamente, la Procuraduría es menos conocida donde precisamente es más necesaria: entre los más débiles y vulnerables de la sociedad salvadoreña. El que el 60 por ciento de los campesinos o que el 66 por ciento de los analfabetas desconocen a la Procuraduría habla, en cierta forma, de la poca presencia que ha tenido esta institución en los sectores más necesitados, en sus primeros tres años de existencia. Sin embargo, a la hora de ser evaluada, la gente que conoce a la Procuraduría 
tiende a expresar una postura positiva sobre su desempeño. De hecho, más de la mitad de los encuestados piensa que el trabajo que ha realizado la institución de derechos humanos es bueno o muy bueno, el 27 por ciento califica ese trabajo como regular y sólo el 10 por ciento piensa que su labor ha sido mala o muy mala. Contrariamente a lo esperado, la Procuraduría es mejor evaluada en los sectores más pobres, donde también existe la mayor proporción de desconocimiento sobre su trabajo; mientras que las opiniones negativas se encuentran con mayor frecuencia entre las personas de los estratos medios y altos.

El sondeo de opinión pública no sólo permitió la evaluación general del desempeño de la Procuraduría sino que también, intentando profundizar un poco en tal evaluación, dedicó otros reactivos para que la población diera su juicio acerca del trabajo de la institución en rubros específicos (ver el Cuadro 7). Una de las cosas que más saltan a la vista de los resultados obtenidos con esta evaluación es que la institución es mejor evaluada de manera general que en cualesquiera de los aspectos que se sometieron a consideración de la ciudadanía, aunque el balance es positivo en todos los rubros. Las áreas en las que la Procuraduría para la Defensa de los Derechos Humanos obtuvo opiniones positivas más frecuentemente son la capacidad y la voluntad para servir y los anuncios en la televisión y la radio. Esto es lógico si se considera que la Procuraduría invirtió muchos de sus recursos en darse a conocer en los medios de comunicación social salvadoreños a través de la publicidad. Por el otro lado de la moneda, la Procuraduría resultó peor evaluada en el área de educación de la ciudadanía en derechos humanos. En el fondo, cerca del 30 por ciento de la población sostiene la idea de que la Procuraduría no hizo los esfuerzos suficientes para educar a la población en el tema de los derechos humanos.

En otro orden, hay que recordar que para mediados de marzo, la asamblea legislativa aún estaba discutiendo sobre la elección de la persona que ocuparía la dirección de la Procuraduría. En un afán por colaborar con el futuro desempeño del funcionario y de la institución, uno de los principales objetivos de esta encuesta era el de precisar, en base a la opinión de los salvadoreños, los retos o tareas más importantes que debería enfrentar quien finalmente resultó seleccionada como directora de la Procuraduría. La población propuso a la nueva directora tres grandes tareas entre otras: velar por los derechos de todos, ser más enérgica y combatir la delincuencia.

\section{Cuadro 7}

Evaluación sobre el desempeño de la Procuraduría para la Defensa de los Derechos Humanos (En porcentajes)

\begin{tabular}{|c|c|c|c|c|}
\hline \multirow[b]{2}{*}{ Rubro evaluado } & \multicolumn{3}{|c|}{ Evaluación } & \multirow[b]{2}{*}{ No sabe } \\
\hline & Negativa & Regular & Positiva & \\
\hline Evaluación general por su desempeño hasta la fecha! & 10.1 & 27.1 & 57.3 & 5.5 \\
\hline La investigación en violaciones de derechos humanos2 & 19.1 & 22.6 & 51.5 & 6.8 \\
\hline La educación de la gente sobre los derechos humanos & 29.3 & 21.1 & 42.9 & 6.6 \\
\hline La actuación en situaciones especiales & 17.5 & 20.8 & 46.7 & 15.0 \\
\hline La capacidad y la voluntad de servir & 11.7 & 23.2 & 58.0 & 7.1 \\
\hline Sus anuncios en la televisión y en la radio & 11.5 & 19.4 & 59.6 & 9.5 \\
\hline La firmeza del Procurador en las situaciones de conflicto & 18.6 & 21.3 & 40.9 & 19.2 \\
\hline \multicolumn{5}{|c|}{$\begin{array}{l}\text { ¿Cómo evaluaría Ud. a la Procuraduría para la Defensa de los Derechos Humanos por su desempeño hasta la } \\
\text { fecha? } \\
\text { ¿Cómo evalúa el desempeño de la Procuraduría en los siguientes aspectos? [Excelente, bueno, regular, malo o } \\
\text { pésimo.] }\end{array}$} \\
\hline
\end{tabular}




\section{Las principales tareas de la Procuradora de derechos humanos}

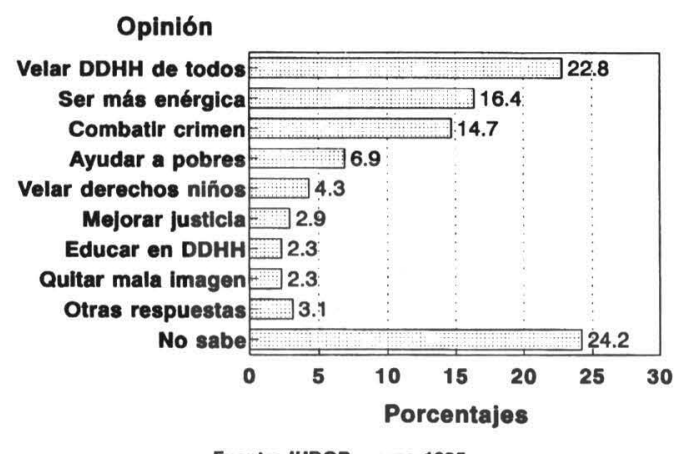

Fuente: IUDOP, marzo 1995.

Figura 2

Dos de las respuestas principales llaman particularmente la atención y ameritan un comentario. Cuando la población pide que la nueva Procuradora sea más enérgica, de alguna forma buscan señalar la necesidad de levantar el perfil de actuación que hasta ahora ha tenido la figura del procurador. No es casual encontrar esta respuesta dado que el antiguo funcionario fue muchas veces acusado de no ser lo suficientemente enérgico como para enfrentar las situaciones especiales. Por otro lado, una porción significativa de los encuestados propuso como tarea esencial en derechos humanos combatir la delincuencia. De nuevo, vuelve a presentarse la vinculación entre derechos humanos y delincuencia. Y es que la gente asocia directamente y de forma errónea la presencia de la violencia con el irrespeto de los derechos humanos.

Finalmente, los salvadoreños también tuvieron la oportunidad de expresar las cualidades personales que debería tener la nueva funcionaria de derechos humanos. El 34 por ciento de los consultados afirmó que la Procuradora debe ser honesta u honrada, el 11.3 por ciento opinó que debe ser una persona que ayude a la gente pobre, el 10.9 por ciento manifestó el deseo de que fuese una persona neutral, el 9.1 por ciento aludió a la capacidad, el 8.6 por ciento pidió responsabilidad a la nueva funcionaria, el 6.3 por ciento demandó firmeza y el 5.6 por ciento de los encuestados mencionó la justicia como cualidad de la funcionaria. Cerca del
14 por ciento de los consultados no dio su opinión sobre este tema. Honestidad y honradez son características mejor apreciadas entre las personas de los sectores altos y de formación superior, empatía para ayudar a la gente pobre es una cualidad que fue más citada por los sectores medios urbanos, al igual que la apoliticidad y la capacidad.

\subsection{La Corte Suprema de Justicia}

Para un poco más de la mitad de la población (el 52 por ciento), la Corte Suprema de Justicia no se preocupa por proteger los derechos humanos de todos los salvadoreños. Como puede verse en el Cuadro 8, esta postura es más frecuente entre los salvadoreños de clase media y entre las personas con mayor instrucción académica que entre el resto de ciudadanos.

\section{Cuadro 8}

Opinión de si la Corte Suprema de Justicia se preocupa por proteger los derechos humanos de todos los salvadoreños según sector social y nivel educativo

(En porcentajes)

\begin{tabular}{|c|c|c|c|}
\hline \multirow[b]{2}{*}{ Variables } & \multicolumn{2}{|c|}{ Opinión } & \multirow[b]{2}{*}{ No sab } \\
\hline & No & Sí & \\
\hline \multicolumn{4}{|l|}{ Estrato } \\
\hline Alto & 50.0 & 37.2 & 12.8 \\
\hline Medio-alto & 65.4 & 28.3 & 6.3 \\
\hline Medio-bajo & 67.2 & 23.6 & 9.2 \\
\hline Obrero & 58.3 & 28.9 & 12.8 \\
\hline Marginal & 40.9 & 42.9 & 16.2 \\
\hline Rural & 42.5 & 34.2 & 23.2 \\
\hline \multicolumn{4}{|l|}{ Nivel educativo } \\
\hline Ninguno & 39.2 & 32.5 & 28.2 \\
\hline Primaria & 42.8 & 35.4 & 21.8 \\
\hline Plan básico & 55.2 & 32.6 & 12.2 \\
\hline Bachillerato & 64.4 & 27.4 & 8.2 \\
\hline Superior & 63.1 & 30.9 & 6.0 \\
\hline Todos & 52.0 & 32.4 & 15.7 \\
\hline
\end{tabular}

¿Cree Ud. que la Corte Suprema de Justicia se preocupa por proteger los derechos humanos de todos los salvadoreños? 
Sin embargo, cuando se les preguntó a los consultados sobre el desempeño de la Corte Suprema de Justicia, la evaluación general arrojó un resultado relativamente positivo. El 34 por ciento de los salvadoreños piensa que el trabajo de la Corte ha sido bueno o excelente, el 31 por ciento opina que ha sido regular, mientras que el 18.3 por ciento sostiene que ha sido malo o pésimo; el 16.7 por ciento dijo no tener opinión al respecto.

Estos datos estarían indicando que, a los ojos de la población, el trabajo de la Corte en derechos humanos no muestra el mismo nivel de empeño que en otras áreas. Esto, ciertamente, refleja el perfil que ha mostrado la Corte Suprema de Justicia en el rubro de los derechos humanos. De hecho, en la actualidad pareciera prevalecer la idea de que el problema de los derechos humanos es cuestión de la Procuraduría y no es un asunto de otras instituciones encargadas de la administración de justicia.

\subsection{La Policía Nacional Civil}

El manejo del tema de los derechos humanos por parte de la Policía Nacional Civil no ha resultado ser una situación fácil para esta institución de seguridad pública. No son pocas las denuncias de irrespeto a los derechos fundamentales que la Policía Nacional Civil ha tenido que afrontar en su corta existencia. No obstante, ya se ha visto que un importante segmento de la ciudadanía ha identificado a la nueva policía como una institución que defiende los derechos humanos (ver la Figura 1). El sondeo de marzo preguntó directamente si la Policía Nacional Civil respeta los derechos humanos de la población. Los datos mostraron una opinión más bien dividida acerca este tema. Aunque casi el 40 por ciento (el porcentaje más alto) considera que la Policía Nacional Civil sí respeta los derechos humanos de los salvadoreños, existe un 22 por ciento que opina lo contrario y un 33 por ciento que piensa que la policía respeta los derechos humanos a veces (ver la Figura 3 ).

¿Cómo se explica entonces esta "polarización" de opiniones acerca de la Policía Nacional Civil? Los datos que ofrece esta encuesta vienen apuntando a una estrecha relación en el ideario público entre la delincuencia y la falta de respeto de los derechos humanos. En esta errónea concepción, como ya se adelantó arriba, el papel que juega la Policía Nacional Civil en el combate contra el crimen la ubica como una de las abanderadas de la protección de los derechos humanos de los salvadoreños, pero al mismo tiempo, por algunas actuaciones de conocimiento público, es difícil esperar una opinión más favorable en cuanto a su actitud de respeto de los derechos fundamentales.

\section{¿Cree Ud. que la PNC respeta los derechos humanos de la población?}

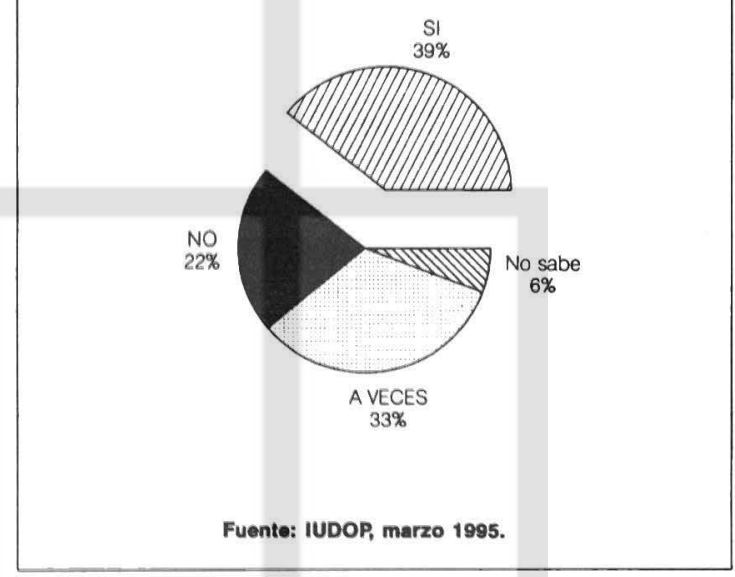

Figura 3

\subsection{ONUSAL}

ONUSAL constituye probablemente una de las instituciones que más efectivamente pudo recoger la confianza de la población, en términos de protección de los derechos humanos. Esto llegó a tales niveles que en sus años de presencia activa en El Salvador inclusive alguna gente acudía a la oficina regional más cercana para buscar dirimir disputas legales que no tenían nada que ver con su mandato.

Llegado el fin de su compleja presencia en El Salvador, la encuesta de marzo indicó que ONUSAL se retiraba con el 51.7 por ciento de opiniones favorables entre la población, frente a un 18 por ciento de juicios negativos y un 24 por ciento de personas que piensa que ha hecho un trabajo regular (se preguntaba de forma general: ¿qué opinión le merece ONUSAL al final de su 
mandato en El Salvador?; las respuestas eran: excelente, buena, regular, mala o pésima). Un dato que merece la pena mencionar es que las opiniones positivas fueron mucho más frecuentes entre los más pobres que entre los ricos. En los sectores marginales, el 61 por ciento evaluó favorablemente a Naciones Unidas.

En estas circunstancias, no es de extrañar que cerca de la mitad de los salvadoreños y sobre todo los más desposeídos sostengan que la situación de respeto de los derechos humanos se agravará después de la salida de la misión de Naciones Unidas. Efectivamente, como muestra el Cuadro 9, buena parte de la población teme que las condiciones de respeto a los derechos fundamentales se deterioren con la salida de ONUSAL.

Hasta aquí los resultados de la encuesta que muestran las opiniones de la población sobre las instituciones que han estado implicadas en la protección de los derechos humanos. El siguiente apartado presenta el panorama general del respeto de los derechos fundamentales desde la opinión pública.

\section{La opinión sobre el respeto de los derechos humanos en El Salvador}

Para 37 de cada cien personas, en El Salvador aún no existe respeto de los derechos humanos, 28 de esas cien personas piensan que existe un poco de respeto y 31 consideran que sí prevalece el respeto de los derechos fundamentales en el país. A pesar de lo equilibrado de las respuestas, éstas reflejan más bien un balance pesimista acerca de las condiciones de los derechos fundamentales. $Y$ es que la misma encuesta reveló que la tercera parte de los ciudadanos salvadoreños piensa que la situación de impunidad sigue igual o se ha incrementado en la sociedad salvadoreña; sólo el 17 por ciento está dispuesto a argumentar que la impunidad ha disminuido o ha terminado $\chi_{\text {Las }}$ principales razones para sostener que la impunidad sigue igual o se ha incrementado son que no se castiga a los culpables, no ha cambiado nada y se ha incrementado la delincuencia; por otro lado, los argumentos para decir que la impunidad ha terminado o disminuido se centran en que se puede ver el cambio, se han investigado los crímenes y hay justicia (ver el Cuadro 10).

En estas respuestas se encuentra que mucho de la valoración sobre la impunidad depende ahora de la situación de la delincuencia imperante en El Salvador. Referencias como que no se castiga los culpables, se ha incrementado la delincuencia, no se aplica la ley o la falta de justicia tienen un gran componente, referido a la criminalidad en el país cuando no se refieren totalmente a ella.

En estas circunstancias, ¿cuáles son, a juicio de los salvadoreños, los derechos que más se violan en la actualidad? Los resultados arrojaron que los derechos más básicos, los civiles y políticos, los de primera generación, son los que más se violan en El Salvador. Estos se presentaron en el si-

\section{Cuadro 9}

Opinión sobre la situación de los derechos humanos después de la salida de ONUSAL, según sector social (En porcentajes)

\begin{tabular}{|c|c|c|c|c|c|c|c|}
\hline \multirow[b]{2}{*}{ Opinión } & \multicolumn{5}{|c|}{ Sector social } & \multirow[b]{2}{*}{ Rural } & \multirow[b]{2}{*}{ Todos } \\
\hline & $\overline{\text { Alto }}$ & Med-al & Med-baj & Obrero & Marginal & & \\
\hline Sí se agravará & 34.6 & 33.1 & 54.0 & 52.1 & 53.2 & 50.4 & 48.7 \\
\hline No se agravará & 60.3 & 57.5 & 36.2 & 32.7 & 24.0 & 24.2 & 33.6 \\
\hline No sabe & 5.1 & 9.4 & 9.8 & 15.2 & 22.7 & 25.4 & 17.7 \\
\hline
\end{tabular}

¿Cree Ud. que después de la salida de ONUSAL, la situación de derechos humanos se agravará o no se agravará? 


\section{Cuadro 10 \\ Opinión sobre la impunidad en El Salvador}

\begin{tabular}{lrr}
\hline $\begin{array}{l}\text { Opinión } \\
\text { Razones }\end{array}$ & $\%$ \\
\hline Ha incrementado & 33.0 & \\
Sigue igual & 35.6 & 27.3 \\
No se castiga a los culpables & & 18.3 \\
No hay cambios, nada ha cambiado & & 14.7 \\
Se ha incrementado la delincuencia & & 10.7 \\
No se aplica la ley & 7.7 \\
Falta de justicia & 6.6 \\
Falta de autoridad competente & 5.1 \\
Existe tráfico de influencias & & 2.8 \\
Otras respuestas & & 6.8 \\
No sabe, no responde & & \\
& & \\
Ha disminuido & 16.2 & 28.4 \\
Terminó & 0.6 & 25.3 \\
Se ve el cambio & & 14.9 \\
Se han investigado mejor los casos & 7.2 \\
Hay justicia & & 3.1 \\
Tenemos mejores autoridades & & 21.1 \\
Otras respuestas & & \\
No sabe, no responde & & \\
\hline
\end{tabular}

guiente orden como derechos más violados: derecho a la vida, 22.4 por ciento; derechos de los niños, 15.4 por ciento; derecho a la integridad física, 8.6 por ciento; derecho a la libertad, 8 por ciento; derecho a la seguridad, 7.3 por ciento; derecho al trabajo, 5.6 por ciento; derechos de la mujer, 5.2 por ciento; y otros derechos, 11.4 por ciento. El 16.2 por ciento de los encuestados no mencionó ningún derecho. Si se intentara elaborar un perfil de la situación de los derechos fundamentales en El Salvador a partir de la elección de los derechos más violados, obviamente el balance resultaría muy negativo. A pesar de los importantes cambios de orden político y social para permitir la construcción de una sociedad menos atentatoria contra los derechos humanos, parece que el camino hacia un régimen de respeto de los derechos de la población es todavía muy largo.

En el mismo sondeo, el IUDOP preguntó a los salvadoreños: para usted, ¿quién viola más los derechos humanos en nuestro país? Las respuestas se muestran en el Cuadro 11. Para buena parte de los salvadoreños los violadores de los derechos humanos son los delincuentes; para otra parte, es el mismo gobierno y las autoridades.

Esto complementa una visión sobre la situación de los derechos humanos que se basa en la problemática de la delincuencia que vive actualmente el país. Ahora bien, desde esta perspectiva, ¿es válido hablar de respeto o irrespeto a los derechos fundamentales si se concluye que el centro de la problemática es la delincuencia o la violencia producto del crimen en la sociedad? En principio, no. Aunque estos datos más bien señalan el nivel de confusión de parte de la población en el tema de los derechos humanos, también apuntan a un punto importante del problema de los derechos humanos en El Salvador. Ciertamente, no se puede 


\section{Cuadro 11}

Opinión sobre quién viola más los derechos humanos

según sector social

(En porcentajes)

\begin{tabular}{|c|c|c|c|c|c|c|c|}
\hline \multirow[b]{2}{*}{ Violadores } & \multicolumn{6}{|c|}{ Sector social } & \multirow[b]{2}{*}{ Todo } \\
\hline & Alto & Med-al & Med-baj & Obrero & Marginal & Rural & \\
\hline Los delincuentes & 42.3 & 39.4 & 32.2 & 44.5 & 38.3 & 41.1 & 39.9 \\
\hline El gobierno & 11.5 & 13.4 & 19.5 & 16.6 & 14.3 & 12.5 & 14.6 \\
\hline Todos & 12.8 & 11.0 & 9.8 & 5.7 & 8.4 & 5.9 & 7.8 \\
\hline Las autoridades & 7.7 & 6.3 & 2.9 & 5.2 & 2.6 & 3.9 & 4.3 \\
\hline Patronos & 3.8 & 7.1 & 5.7 & 5.2 & 4.5 & 1.5 & 4.0 \\
\hline Otros & 7.7 & 11.0 & 12.1 & 7.6 & 5.8 & 5.4 & 7.6 \\
\hline No sabe & 14.1 & 11.8 & 17.8 & 15.2 & 26.0 & 29.8 & 21.8 \\
\hline
\end{tabular}

decir que los delincuentes violan los derechos humanos, pero hay que recordar que la situación de criminalidad que vive el país en estos días es en parte producto de la indiferencia y de la negligencia de las autoridades para erradicar las estructuras de impunidad y para depurar el sistema de justicia. En cierta forma, la violencia generada por el crimen es un problema de derechos humanos en la medida en que el Estado no hace lo necesario para que impere la justicia en el sistema social, en la medida en que permite $o$, en ocasiones, promueve la corrupción en las instancias donde se imparte justicia.

Pero volviendo al hecho de que la gente ubica a la delincuencia como el punto central del proble- ma de los derechos humanos, hay que recalcar que este resultado revela el bajo nivel de educación en derechos humanos que poseen los salvadoreños; en tal sentido, es muy actual la petición de algunos ciudadanos para que se eduque a los salvadoreños en el respeto de los derechos fundamentales.

Efectivamente, preguntados los salvadoreños sobre las cosas que se deben hacer para que mejore el respeto de los derechos humanos en el país, las respuestas más frecuentes fueron: combatir la delincuencia, educar a la gente en derechos humanos y crear fuentes de trabajo. Un porcentaje nada despreciable aludió al fortalecimiento de las instituciones relacionadas con la administración de justicia en el país.

\section{Cuadro 12}

Opinión sobre lo que se debe hacer para que la situación de derechos humanos mejore en el país, según sector social

(En porcentajes)

\begin{tabular}{|c|c|c|c|c|c|c|c|}
\hline \multirow[b]{2}{*}{ Medidas } & \multicolumn{5}{|c|}{ Sector social } & \multirow[b]{2}{*}{ Rural } & \multirow[b]{2}{*}{ Todos } \\
\hline & Alto & Med-al & Med-baj & Obrero & Marginal & & \\
\hline Combatir crimen & 37.2 & 33.9 & 31.6 & 40.3 & 35.1 & 40.1 & 37.3 \\
\hline Educar en DDHH & 28.2 & 24.4 & 23.6 & 19.4 & 11.0 & 18.1 & 19.6 \\
\hline Crear más trabajo & 7.7 & 16.5 & 15.5 & 19.0 & 18.8 & 15.9 & 16.3 \\
\hline Fortalecer la PDDH & 7.7 & 7.1 & 9.2 & 5.7 & 8.4 & 5.1 & 6.7 \\
\hline Apoyar a la PNC & 3.8 & 3.9 & 4.6 & 3.3 & 9.1 & 6.4 & 5.5 \\
\hline Otras respuestas & 15.4 & 11.8 & 13.8 & 11.4 & 14.2 & 10.0 & 4.4 \\
\hline No sabe & 0.0 & 2.4 & 1.7 & 0.9 & 3.2 & 4.4 & 2.7 \\
\hline
\end{tabular}


Sin duda, el balance de la situación de los derechos humanos no parece el más positivo, y probablemente el dato más significativo para esta conclusión no es sólo que buena parte de la población piense que aún se violan los derechos humanos o que la mayoría vea en la criminalidad un problema de derechos humanos; el indicador más importante de esta evaluación es el que muestra que sólo el 17 por ciento de los salvadoreños está dispuesto ha sostener que la impunidad ha terminado o ha disminuido en la actualidad.

\section{Otros resultados}

En este apartado se presentan los resultados de otros temas abordados en el sondeo: el problema de las manifestaciones y huelgas recientes, el papel de la Fuerza Armada en el combate de la delincuencia y las simpatías políticas de los ciudadanos salvadoreños.

\subsection{La opinión sobre la inestabilidad política}

En el sondeo que se presenta en estas páginas se dedicaron un par de preguntas al tema de la inestabilidad social y política que vive el país en los primeros tres meses del año. Específicamente se preguntó lo siguiente: "En los últimos meses en El Salvador se han producido manifestaciones, huelgas y tomas por parte de distintos grupos. En su opinión, ¿es ésto un intento por desestabilizar al gobierno o se trata de problemas de defensa de los intereses de los trabajadores y desmovilizados?". Tres de cada cuatro consultados piensan que tales sucesos son producto de la defensa de los intereses de los trabajadores y desmovilizados. Sólo una pequeña porción de los encuestados defendió la tesis de desestabilización. Un análisis por sectores sociales mostró que esta es una idea que tiene mucho arraigo entre las personas que ocupan los sectores socioeconómicamente altos de la población. A quienes opinaron que las huelgas y manifestaciones son un intento por desestabilizar al gobierno se les preguntó: "¿quiénes cree usted que estarían tratando de desestabilizar al país?". Los resultados se muestran en el Cuadro 13. Este indica que quienes piensan en la desestabilización atribuyen la responsabilidad a la izquierda, a ADEFAES, a los sindicatos y a los partidos políti- cos, entre otros; pero cerca del 40 por ciento dijo no saber quién es el responsable.

\section{Cuadro 13}

Opinión sobre las últimas huelgas, manifestaciones y tomas

Opinión \%

Defensa de intereses de trabajadores

y desmovilizados

Intento de desestabilización

$\begin{array}{lr}\text { Responsables } & \% \\ \text { Izquierda } & 11.2 \\ \text { ADEFAES } & 9.0 \\ \text { Gente contra el gobierno } & 8.3 \\ \text { Sindicatos } & 7.9 \\ \text { Partidos políticos } & 4.3 \\ \text { Izquierda y derecha } & 4.0 \\ \text { Otros responsables } & 12.3 \\ \text { No sabe } & 43.0\end{array}$

\subsection{La Fuerza Armada y el combate a la delin- cuencia}

Como una forma de responder al problema de la delincuencia, el gobierno anunció en marzo su intención de ocupar al ejército en el combate contra la criminalidad. Esta medida generó una serie de debates en la sociedad política, pero parecía claro que esta disposición tenía el apoyo de la mayoría de los salvadoreños. El IUDOP decidió incluir este tema en su encuesta de marzo y preguntar las razones por las cuales la población salvadoreña apoyaría o no apoyaría la salida de la Fuerza Armada a las calles para combatir la delincuencia.

Efectivamente, seis de cada diez ciudadanos se mostraban a favor de que el ejército salvadoreño saliera a patrullar las calles para detener el auge delincuencial, tres se declaraban en contra y uno se mantenía sin emitir opinión. La opinión a favor del papel del ejército en la lucha contra la criminalidad se presentó con mayor frecuencia en la medida en que se encuestaba a personas de mayores ingresos; sin embargo, entre las personas de clase 
media baja, el apoyo a esta opinión era sensiblemente menor. Quienes piensan que los patrullajes del ejército serán eficaces para disminuir la criminalidad argumentan que la presencia de los soldados dará mayor seguridad a la ciudadanía, citan también el temor que los ladrones tendrían de la institución armada; otros consideran que el ejército ayudará a la Policía Nacional Civil e inclusive una proporción no despreciable de consultados manifestó que es bueno porque la Fuerza Armada eliminaría (físicamente) a los delincuentes. Por el otro lado, entre el 28 por ciento de ciudadanos que opinaron que los patrullajes del ejército no serían buenos para disuadir a los delincuentes prevalecieron razones como las siguientes: no son capaces, no se puede hacer nada contra la delincuencia, ellos (los soldados) son los ladrones, es volver a lo de antes, etc.

\section{Cuadro 14}

Opinión sobre los patrullajes de la Fuerza Armada para combatir la delincuencia

\begin{tabular}{lrr}
\hline $\begin{array}{l}\text { Opinión } \\
\text { Razones }\end{array}$ & $\%$ & \\
\hline Sí serán eficaces & & 61.6 \\
$\quad$ Dan mayor seguridad & 25.8 & \\
Los ladrones les temen & 21.5 & \\
Son capaces & 17.2 & \\
Ayudarán a la PNC & 15.6 & \\
Es bueno que los eliminen & 13.5 & \\
Otras respuestas & 1.4 & \\
No sabe, no responde & 4.9 & \\
& & \\
No serán eficaces & & 28.4 \\
$\quad$ No son capaces & 26.9 & \\
No se puede hacer nada & 23.9 & \\
Ellos son los ladrones & 19.3 & \\
Es volver a los de antes & 16.8 & \\
Otras respuestas & 7.9 & \\
No sabe, no responde & 5.2 & \\
& & \\
No sabe & & 10.1 \\
\hline
\end{tabular}

Los datos anteriores estarían mostrando un virtual apoyo de la población a que la Fuerza Armada desempeñe el papel de gendarme; sin em- bargo, la consulta del IUDOP se amplió también al campo de la política: "¿en su opinión, los militares deben o no deben meterse en política?". A pesar de que más de la mitad de los salvadoreños apoyaba la salida del ejército a las calles, casi tres cuartas partes de la población (73.3 por ciento) consideran que los militares no deben inmiscuirse en la política, sólo el 13.4 por ciento de los consultados pensaba que sí deben meterse en política y un porcentaje igual se abstuvo de opinar. Y es que aparentemente, la situación de delincuencia que percibe la población desde el fin de la guerra ha impulsado a muchos ciudadanos a pedir el retorno del ejército, pero ello no ha implicado que los salvadoreños aprueben la intromisión de la Fuerza Armada en todas las esferas de la vida nacional y sobre todo en la política, donde ha jugado un papel protagónico hasta hace poco tiempo.

\subsection{Las preferencias partidistas}

Probablemente, el dato más relevante de los resultados de la pregunta por preferencia política es el que indica que cerca de un 60 por ciento de los salvadoreños no tiene partido de preferencia en la actualidad. S6́lo el 37 por ciento de los ciudadanos tendría un partido favorito si las elecciones se hubiesen realizado en marzo de 1995: ARENA, FMLN y Partido Demócrata Cristiano mantendrían ciertas cuotas de simpatía, pero con un sensible desgaste respecto al apoyo que recibieron en las elecciones del año pasado.

\section{Partido por el que votaría si las elecciones} fueran el próximo domingo

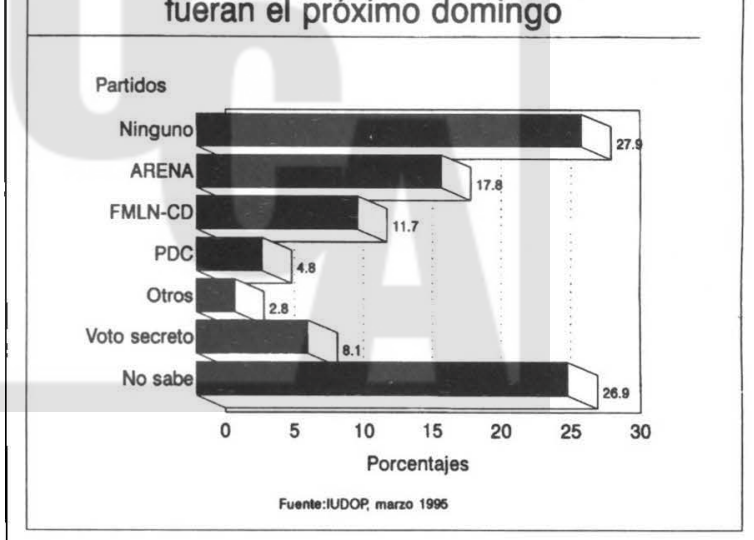

Figura 4 


\section{A manera de conclusión}

En términos generales, la encuesta de marzo del IUDOP revela el poco conocimiento que tiene la población sobre el tema de los derechos humanos, acompañada de una confusión bastante frecuente entre el problema de la violencia y el respeto de los derechos humanos. Sin embargo, es claro que la situación de criminalidad por la que pasa El Salvador en la actualidad tiene parte de sus raíces en la impotencia del Estado para combatir las estructuras de impunidad que violaban los derechos humanos durante la guerra y aún ahora.

En tal sentido, las peticiones de los ciudadanos de combatir la delincuencia como una forma de resolver el problema de los derechos humanos, pasa necesariemante por el compromiso de las instituciones encargadas de la administración de justicia en el país para erradicar los esquemas de impunidad que aún persisten en las actividades de ciertos grupos. Por otro lado, es claro también que uno de los grandes retos de tales instituciones $y$, sobre todo de la Procuraduría, tiene que ver con la educación y la formación de los salvadoreños en el tema de los derechos humanos. Poco o nada se puede lograr en este país, si a la par de importantes reformas legales que protejan más a la población, no se educa a la misma en los derechos fundamentales de todos. 